\title{
Comparing the impact of vaccination strategies on spread of COVID-19, including a novel household-targeted vaccination strategy
}

\author{
André Voigt ${ }^{1 *}$, Stig Omholt ${ }^{2}$, Eivind Almaas ${ }^{1,3}$ \\ 1 Department of Biotechnology and Food Science, NTNU - Norwegian University of \\ Science and Technology, Trondheim, Norway \\ 2 Department of Circulation and Medical Imaging, NTNU - Norwegian University of \\ Science and Technology \\ 3 K.G. Jebsen Center for Genetic Epidemiology, NTNU - Norwegian University of \\ Science and Technology \\ *Correspondence: andre.voigt@ntnu.no
}

\begin{abstract}
With limited availability of vaccines, an efficient use of the limited supply of vaccines in order to achieve herd immunity will be an important tool to combat the wide-spread prevalence of COVID-19. Here, we compare a selection of strategies for vaccine distribution, including a novel targeted vaccination approach (EHR) that provides a noticeable increase in vaccine impact on disease spread compared to age-prioritized and random selection vaccination schemes. Using high-fidelity individual-based computer simulations with Oslo, Norway as an example, we find that for a community reproductive number in a setting where the base pre-vaccination reproduction number $R=2.1$ without population immunity, the EHR method reaches herd immunity at $48 \%$ of the population vaccinated with $90 \%$ efficiency, whereas the common age-prioritized approach needs $89 \%$, and a population-wide random selection approach requires $61 \%$. We find that age-based strategies have a substantially weaker impact on epidemic spread and struggle to achieve herd immunity under the majority of conditions. Furthermore, the vaccination of minors is essential to achieving herd immunity, even for ideal vaccines providing $100 \%$ protection.
\end{abstract}

\section{Introduction}

Population-wide vaccination is the most promising approach for long-term COVID-19 disease management [1]. Due to limited vaccine availability, in particular in the early stages of deployment, most authorities have to choose between vaccination prioritization schemes, aiming for a balance between total mortality, years of life lost, and cumulative levels of infections.

In responding to the COVID-19 pandemic, many countries have opted for a strategy of suppressing infection rates (with varying success) through stringent social, work and travel restrictions until a sufficient amount of vaccines are deployed to adequately mitigate the public health risks of COVID-19. There are two criteria which individually satisfy the latter objective. The first is achieving comprehensive vaccine coverage for those groups at a non-acceptable risk of death or severe illness. In this case, lifting restrictions may still lead to an increase in disease prevalence but with limited adverse 
public health effects. The second is by sufficiently inhibiting transmission that the reproductive number of the disease drops below unity, preventing exponential growth (also known as herd immunity), thus protecting vulnerable individuals from illness by limiting their risk of exposure.

In most European countries and the US, health care workers and the elderly were initially prioritized to receive vaccinations in order to reduce mortality and maintain health service readiness $[2,3$. However, in areas where prevalence is very low, any reduction in mortality rate would translate into limited gains in terms of prevented deaths. In such conditions, a strategy that more effectively targets case prevention may be a more efficient approach, even if it has a lesser impact in terms of per-case risk of serious illness and/or death. Where this is the case, as vaccines not only prevent infection but also may significantly inhibit transmission [4, one should identify vaccination strategies that bring the reproductive number $R$ below unity with the least vaccination effort, as this would enable stable long-term suppression of cases to near zero.

The main objective of this article is to compare the effect of different vaccination strategies on epidemic spread and their ability to achieve herd immunity, even as disease control measures are lifted. As the exact effect on social contact (and consequently R) of lifting current measures is unknown, we opt for an incremental approach, where we gradually increase the amount of contact in the network's random layer, simulating increased activity as a result of lifting of restrictions. By varying the amount of random contact, we can therefore create a set of baselines, for which we can compute $R_{0}$ assuming there is no immunity in the population. For each baseline, we can then produce a set of vaccine distributions defined by the amount of individuals fully vaccinated (as a fraction of the population) and the strategy used to select individuals for vaccination.

Multiple variants of the SARS-CoV-2 virus are currently in circulation world wide. The initial Wuhan strain for which the vaccines were developed, has a markedly lower potential for transmission than several later strains. During spring and summer of 2021, the two strains that dominate global infections are the B.1.1.7 (Alpha) and B.1.617.2 (Delta) strains. The Alpha strain is estimated to be $40-80 \%$ more transmissible than the original strain 57 that spread world-wide, whereas the Delta strain is considered to be 40-60\% more transmissible than the Alpha strain. In this study, we report results for both Alpha and Delta, since their transmission properties may affect the efficacy of vaccination strategies that intend to curb spread.

\section{Materials and methods}

\section{Computational model}

The results in this article were obtained through the use of a multi-layer individual based epidemic model, previously described in [8]. Briefly, the model represents daily spread and progress of COVID-19 for a city with demographics similar to Oslo, Norway (approx. 700,000 inhabitants). Each inhabitant is represented as a discrete entity defined by personal attributes (age, COVID-19 state, and history). Each individual is then assigned to one or more cliques, through which it comes into contact with other individuals. These cliques represent interactions which are repeated over an extended period of time, such as households, workplace contact among colleagues, schools, child daycare and elder care facilities. In addition to these cliques, for each new day, each individual also comes into contact with a random group of individuals chosen from the entirety of the municipal population.

The disease dynamics in the model follow a typical extended SEIR model, in which 

susceptible individual transitions to the exposed state. An exposed individual will, after a period of time, progress to either an asymptomatic or a pre-symptomatic state. The first state corresponds to those infections for which symptoms never manifest and will transition next to the recovered state. Pre-symptomatic individuals will at some point develop symptoms, with a further possibility of hospitalization and/or death. As an individual transitions to any state except recovered or dead, the time spent in the new state as well as the following transition to be made is chosen at random according to a parameter table described in Ref. [8]. Recovery is assumed to provide immunity to further re-infection for at least the duration of the simulation.

For a given parameter set (disease infectiousness, disease control measures, and vaccinated population), epidemic spread on the model will follow a steady-state exponential growth described by a constant reproduction number $R$ as long as infection-induced immunity remains at a low level. Due to the multi-layer dynamics involved, a sudden change in parameters will lead to a transitional period of about 10 days until $R$ stabilizes at the steady-state for the new parameter set, as described in Ref. 8] (Supplementary material).

\section{Simulation process and computing $R$}

In order to estimate $R$ for a given combination of social contact and vaccine distribution, we perform simulations in a three-step protocol intended to represent COVID-19's ability to spread from a few localized clusters. The first step of model initialization consists of the following two tasks:

1. 20 randomly selected individuals are infected as seed cases. No individuals are vaccinated at this point.

2. Infection is allowed to spread from the seed cases at an accelerated rate (representing a combination of free local spread and case importation) until the system reaches 100 symptomatic cases. No vaccinations or other infection control measures are in place at this point.

As the first task infects all the seed cases at the same time, the number of cases will briefly dip after about one generation (9 days) as most of the initial cases recover within a few days from each other. This effect dissipates within two generations. The purpose of the second task is to allow time for this to happen.

The model is then adjusted to the desired amount of random contact, and the population is simultaneously and suddenly immunized according to the chosen vaccination strategy and capacity. Our simulations assume a hypothetical single-dose vaccine with a specified efficiency, which represents the proportion of vaccinated individuals effectively immunized by the vaccine.

This approach represents a substantial simplification of real-world vaccination deployments, which gradually take place and typically revolve around multiple types of vaccines with varying degrees of efficiency 9 11, and many of which only provide partial protection after a single dose and require a second dose, administered some time after the first, in order to provide maximal protection. Nonetheless, the modelling approach of simultaneous vaccination of a specified fraction of the population can be seen as representing a "snapshot" of a gradual deployment at a given point in time. Consequently, varying vaccination fractions for a given strategy can be interpreted as different time points in a gradual vaccination deployment. 
With the specified fraction of the population vaccinated and amount of random contact set to the desired level, the model runs for 40 (in-model) days. We then compute an individual reproductive number $R$ for each person that has recovered before the 40-day mark by simply directly counting the number of secondary infections caused by that person over the course of their illness. For a given day, we define a daily $R$ as the average of individual reproductive numbers for all persons that are sick on that day (including secondary infections caused by each individual on other days). In reporting a final $R$ for a given combination of activity and vaccination, we take the average of daily $\mathrm{R}$-values from days 12 to 17 of the 40 days in this second step.

The first 11-day interval serves to pass the transient phase between change of contact activities from the initialization step, mentioned in the last paragraph of the section titled "computational model" above. The 23 day-gap after day 17 serves to ensure that all persons infected up to day 17 have had time to recover, in order to get an accurate assessment of the number of secondary infections caused by all persons counted on that day. An insufficient gap would mean that the subset of infected persons that have time to recover (which are the only ones for which we can count an accurate individual reproduction number) would be biased towards those with shorter illnesses, thus would have less time to cause secondary infections. This would cause an underestimation of the actual average $R$.

\section{Vaccination strategies}

For the purposes of this article, we consider four vaccination strategies: A random approach where all individuals are equally likely to be selected for vaccination (regardless of age or other characteristics), one where selection is according to decreasing age (the eldest are vaccinated first), one in which adults (18 and above) are selected by increasing age, and a novel strategy we call "effective household reduction" (EHR) which we describe below in more detail. The motivation for the EHR strategy comes from the observation that a substantial share of COVID-19 infections happen between individuals in the same household [12], and an observed association between the number of large households and the prevalence of COVID-19 8, 13. EHR aims to disrupt infection by targeting large households as possible infection hubs. In summary, EHR can be described as allocating each marginal vaccination by choosing the oldest individual from one of the households with the largest number of unvaccinated members.

As an example, consider a hypothetical community of 5 households labeled A through E, with 5 individuals in household A, 4 in household B, 3 in C, 2 in D, and 1 in E. This gives a total of 15 individuals, none of which are previously immune. Suppose that we only have the capacity to fully vaccinate 5 individuals. In line with the reasoning above, EHR gives the following vaccination strategy:

1. The largest household by effective size is A, with 5 individuals. The first vaccine is therefore allocated to a member of A, with the eldest member chosen.

2. A and B are now tied in terms of effective size, with 4 susceptible individuals each. We therefore vaccinate one person each from both $\mathrm{A}$ and $\mathrm{B}$.

3. A, B, and C are now tied in terms of effective size, with 3 individuals each. With enough capacity left to vaccinate only two people, we therefore randomly choose two of the three households, and vaccinate one individual in each of the randomly chosen households. 


\begin{tabular}{|c|c|c|c|c|c|c|c|}
\hline & \multicolumn{6}{|c|}{ Reproductive number $R$} \\
\hline & & \multicolumn{3}{|c|}{$70 \%$ effective } & \multicolumn{3}{|c|}{$90 \%$ effective } \\
\hline & Vaccinated & Random & EHR & Decreasing age & Random & EHR & Decreasing age \\
\hline \multirow{3}{*}{$\frac{\pi}{\tilde{Z}}$} & \multirow{3}{*}{$\begin{array}{l}20 \% \\
50 \% \\
80 \%\end{array}$} & 2.10 & 1.99 & 2.28 & 1.99 & 1.83 & 2.23 \\
\hline & & 1.70 & 1.52 & 2.00 & 1.46 & 1.23 & 1.88 \\
\hline & & 1.18 & 1.16 & 1.67 & 0.78 & 0.79 & 1.49 \\
\hline \multirow{3}{*}{$\frac{\pi}{\stackrel{\pi}{ \pm}}$} & \multirow{3}{*}{$\begin{array}{l}20 \% \\
50 \% \\
80 \%\end{array}$} & 2.52 & 2.39 & 2.69 & 2.40 & 2.22 & 2.61 \\
\hline & & 2.05 & 1.88 & 2.32 & 1.77 & 1.52 & 2.16 \\
\hline & & 1.44 & 1.39 & 1.85 & 0.97 & 0.93 & 1.64 \\
\hline
\end{tabular}

Table 1. Effect of vaccine deployment on $R$, the reproductive number, in response to vaccination strategies for COVID-19 Alpha and Delta variants. Epidemic reproduction number as a function of vaccinated fraction of population and vaccine efficiency for each of the four presented strategies, assuming a base reproduction number of $R_{0}=2.5$ for the COVID-19 Alpha variant and $R_{0}=3$ for the Delta variant.

\section{Materials}

All code used for this article is available at dx.doi.org/10.6084/m9.figshare.15178386. Developments to the underlying model are available at https://github.com/andrevo/covid19-ntnu.

\section{Results}

The central premise of our proposed vaccine strategy of "effective household reduction" (EHR), is that a household's effective size from an epidemic point of view is equal to the number of susceptible people in that household. This effective size is the actual number of people minus the number of immune people in said household, as immune individuals are essentially non-existent in the context of an epidemic. It may be tempting to target household infections by vaccinating all individuals in the largest households, since an individual's ability to spread infection within their household increases with the number of susceptible individuals. However, as soon as a single individual is vaccinated, the effective household size falls by one when assuming ideal protection, thus reducing the relative benefit of vaccinating the remaining household members compared to the first individual.

Comparing the presented strategies (Fig. 1), we see that for both the COVID-19 Alpha and Delta variants, EHR consistently outperforms the other three strategies with regards to reduction of spread (in our scenario assuming $R_{0}=3$ ), random vaccination comes second, followed by vaccination by increasing age and vaccination by decreasing age coming last. Here, we will use the notation $R(S, F, E)$ to denote the reproductive number in a population with with a fraction $F$ vaccinated according to strategy $S$. For instance, $R(\mathrm{EHR}, 20 \%, 90 \%)$ is the reproductive number in a population with $20 \%$ of the population vaccinated with $90 \%$ efficiency according to the EHR strategy. Comparing EHR to the other strategies, we find that the gap increases with the vaccinated fraction of the population until about $50 \%$, with $R(\mathrm{EHR}, 50 \%, 100 \%)=1.34$ vs $R($ Random, $50 \%, 100 \%)=1.62$, with slightly smaller gaps at $90 \%$ and $70 \%$ efficiency (in the case of the Delta variant), as reported in Table1. As people get vaccinated past this point, the difference begins to narrow, overlapping (within error bars) at $100 \%$ vaccination coverage (which should trivially be true, as there is only one way to select $100 \%$ of the population). We also note that vaccinating children appears to be an essential requirement in achieving herd immunity, with the adult-only strategy failing to achieve herd immunity even for ideal vaccines providing $100 \%$ protection. Interestingly, 


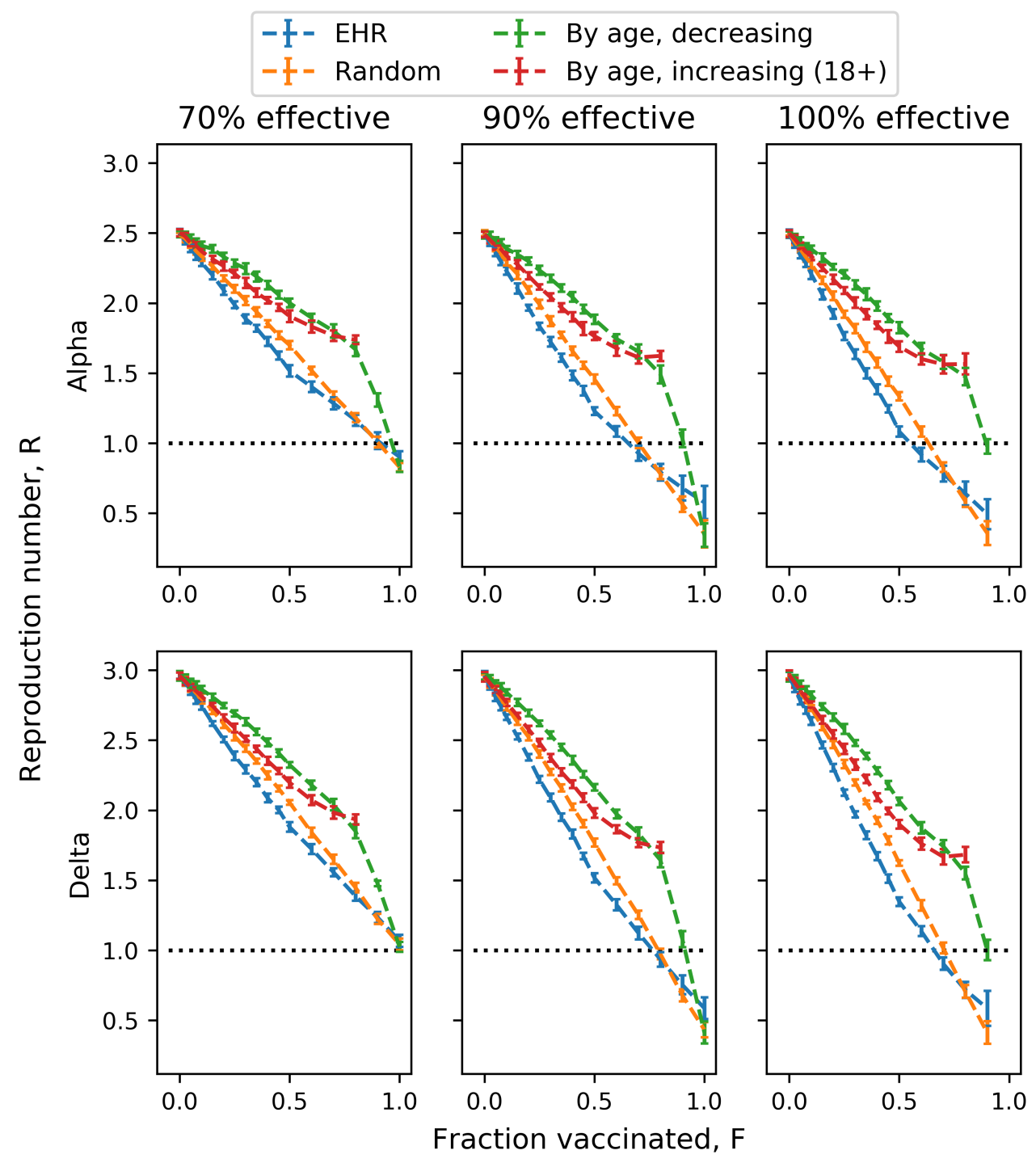

Fig 1. Effect of vaccine deployment on the reproductive number, according to strategy and variant. Epidemic reproduction number as a function of vaccinated fraction of population and vaccine efficiency for each of the four presented strategies, assuming a base reproduction number of $R_{0}=2.5$ for the COVID-19 Alpha variant and $R_{0}=3$ for Delta.

with $70 \%$ vaccine efficiency, none of the strategies achieve herd immunity even at $100 \%$ vaccination coverage. Looking at the intersect between the R-curves and the herd immunity threshold (i.e., $R=1$ ), we find that EHR exhibits a modest advantage compared to random vaccination, with a more substantial edge relative to the elderly-first strategy.

The actual $R_{0}$ for COVID-19 is still subject to substantial uncertainty. Looking at a variety of re-opening scenarios (Fig. 2), we find that EHR and random vaccination perform quite similarly (with a minor edge in the favor of EHR, as seen in Fig. 1), but substantially better than either of the age-based strategies. For lower values of $R_{0}$, EHR achieves herd immunity faster than the random strategy, but this gap narrows as $R_{0}$ 
medRxiv preprint doi: https://doi.org/10.1101/2021.01.30.21250828; this version posted August 19, 2021. The copyright holder for this preprint (which was not certified by peer review) is the author/funder, who has granted medRxiv a license to display the preprint in perpetuity.

\section{It is made available under a CC-BY-NC 4.0 International license .}

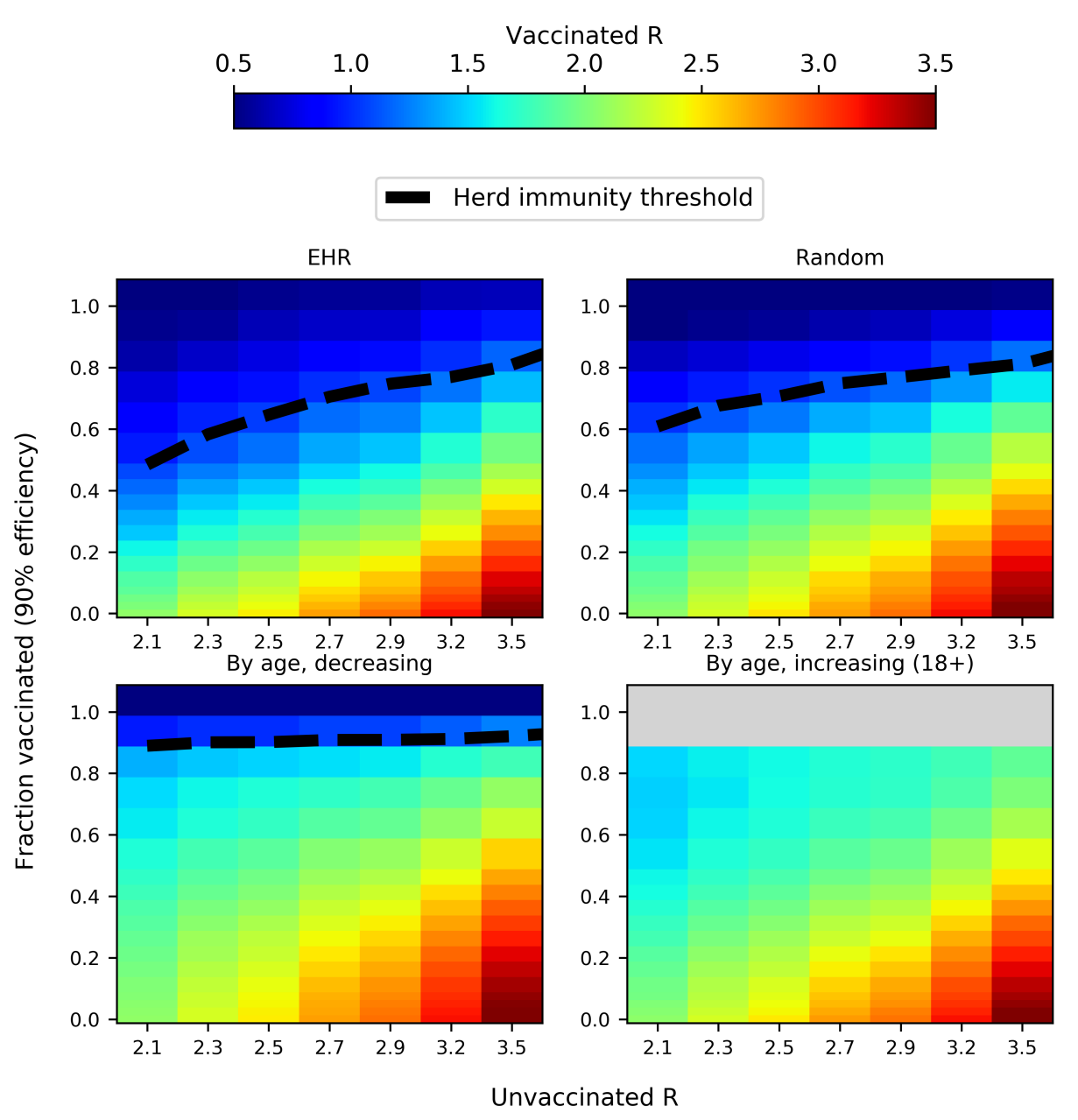

Fig 2. Impact of different vaccination strategies on the reproductive number $R$ according to varying pre-vaccination $R_{0}$. $R_{0}$ is adjusted by varying the amount of contact in the random layer of the model, while other layers (schools, workplaces, households) are kept constant. Dashed lines indicate the fraction of population which needs to be vaccinated in order to achieve herd immunity $R<1$ depending on $R_{0}$ for an unvaccinated population. Herd immunity thresholds are also shown for random and age-dependent vaccination for comparison purposes. Grey denotes infeasible vaccination fractions $(>80 \%)$ for the $18+$ strategy.

increases.

Further investigating herd immunity thresholds for a range of values for $R_{0}$ (Fig. 3 Tab. 2), we find that for most parameter choices, EHR achieves herd immunity with fewer vaccinated individuals than the other presented strategies (and universally, dramatically earlier than age-based strategies). At the most extreme, assuming a $90 \%$ effective vaccine and $R_{0}=2$, EHR achieves herd immunity at just $48 \%$ of the population vaccinated, compared to thresholds of $61 \%$ and $89 \%$ for random and eldest-first strategies. At 100\% efficiency, the relative herd immunity thresholds are $44 \%$ and $55 \%$, for the EHR and random strategies, respectively. The difference narrows as 


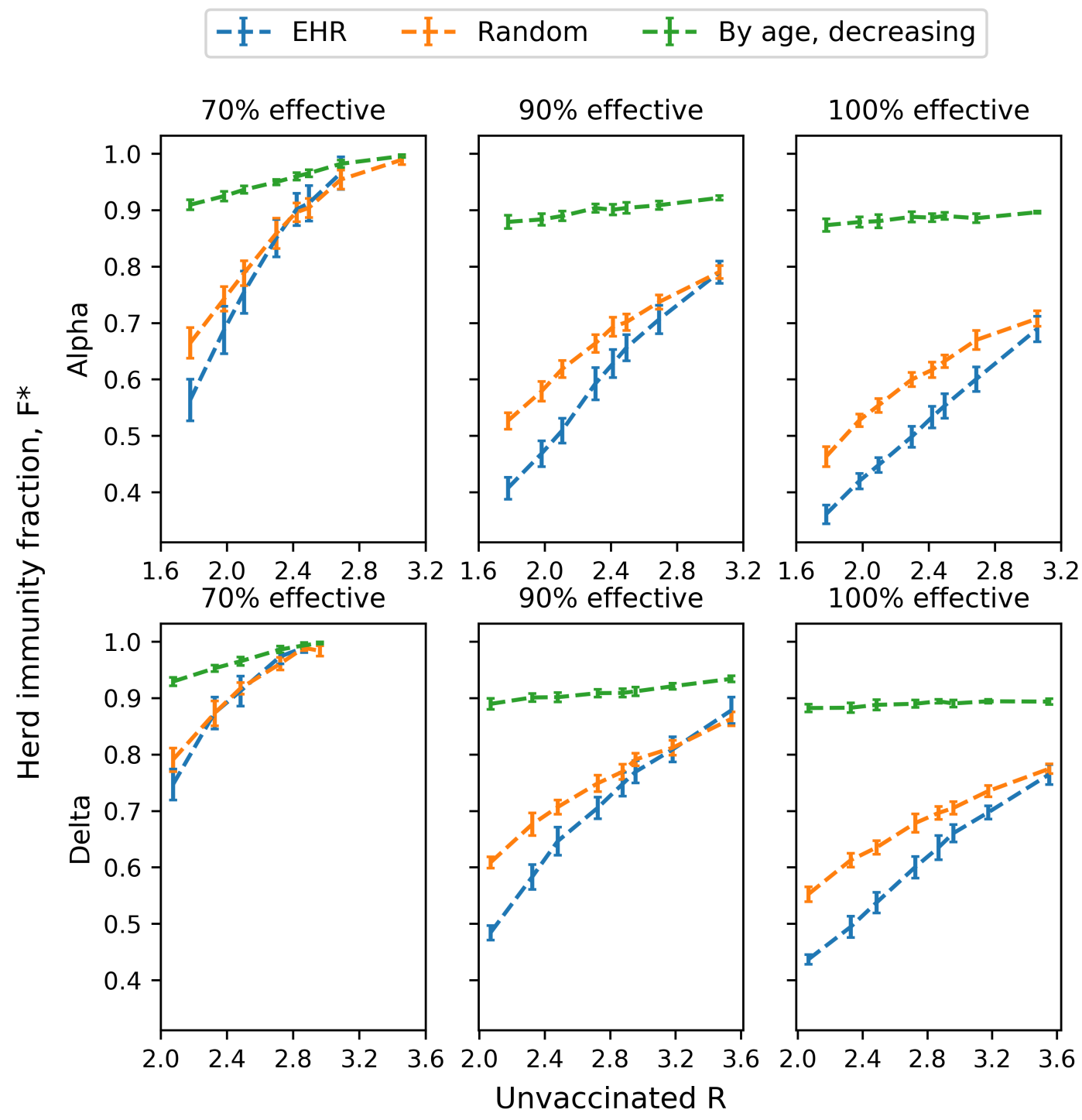

Fig 3. Herd immunity thresholds according to $R_{0}$ and choice of strategy. Fraction of population requiring vaccination in order to reach herd immunity $(R<1)$ according to each strategy and $R_{0}$ for an unvaccinated population.

$R_{0}$ increases, or with less effective vaccines. At $70 \%$ vaccine efficiency, the herd immunity fraction for EHR and random strategies are close to indistinguishable (within error bars) across all values for $R_{0}$. At $90 \%$ effectiveness, the intersection (within error bars) of the EHR and random curves happens for $R_{0} \approx 3$, and at $100 \%$ efficiency, the intersect happens somewhere between $R_{0}=3.2$ and $R_{0}=3.6$.

\section{Discussion}

In general, we find that EHR performs noticeably better than other strategies for lower values of $R_{0}$, while it performs comparable to random vaccination (but still dramatically better than age-based strategies) for higher values of $R_{0}$. While the actual value of $R_{0}$ 


\begin{tabular}{|c|c|c|c|c|c|c|c|}
\hline \multirow{3}{*}{\multicolumn{2}{|c|}{$R_{0}$}} & \multicolumn{6}{|c|}{ Herd immunity threshold, $F^{*}$} \\
\hline & & \multicolumn{3}{|c|}{$70 \%$ effective } & \multicolumn{3}{|c|}{$90 \%$ effective } \\
\hline & & Random & EHR & Decreasing age & Random & EHR & Decreasing age \\
\hline \multirow{3}{*}{$\frac{\overrightarrow{2}}{2}$} & 1.8 & $66.4 \%$ & $56.3 \%$ & $90.9 \%$ & $52.6 \%$ & $40.7 \%$ & $87.9 \%$ \\
\hline & 2.4 & $89.6 \%$ & $90.1 \%$ & $96.0 \%$ & $69.3 \%$ & $62.8 \%$ & $90.1 \%$ \\
\hline & 3.1 & $98.9 \%$ & $>100 \%$ & $99.6 \%$ & $9.0 \%$ & $79.0 \%$ & $92.2 \%$ \\
\hline \multirow{3}{*}{ 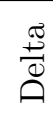 } & 2.1 & $79.1 \%$ & $74.7 \%$ & $92.9 \%$ & $60.8 \%$ & $48.4 \%$ & $89.0 \%$ \\
\hline & 2.7 & $96.1 \%$ & $97.3 \%$ & $98.6 \%$ & $74.9 \%$ & $70.5 \%$ & $90.9 \%$ \\
\hline & 3.5 & $>100 \%$ & $>100 \%$ & $>100 \%$ & $86.3 \%$ & $87.8 \%$ & $93.4 \%$ \\
\hline
\end{tabular}

Table 2. Herd immunity thresholds $F^{*}$ in response to $R_{0}$ and choice of strategy according to strain. Fraction of population requiring vaccination in order to reach herd immunity $(R<1)$ according to each strategy and $R_{0}$ for an unvaccinated population.

for COVID-19 is unknown, it can reasonably be expected to be higher than 3 , particularly for Delta and other recent variants. In this event, the necessary vaccination coverage for random and EHR strategies in order to lift all social restrictions is likely to be close to equal. Nonetheless, intermediate values of $R_{0}$ (between 2 and 3 ) in our model can be interpreted as reflecting partial lifting of general measures on a population-wide level (i.e., measures which affect the population evenly). Both for reasons of economic and social costs as well as due to inherent uncertainties in $R_{0}$, maintaining a maximally strict set of restrictions throughout the vaccination roll-out, which is then suddenly lifted once a critical pre-determined vaccination threshold has been reached, is a difficult proposition. If one instead opts for a gradual lifting of measures as the vaccination coverage increases, an EHR strategy should allow for a somewhat faster lifting of general restrictions as the deployment of vaccines progresses.

Much discussion has been made concerning the role of super-spreaders in the spread of COVID-19. In fact, the EHR strategy fundamentally rests on the notion, common in network-based approaches to studying epidemic spread and immunization 14 17, of targeting heterogeneities in how well individual nodes (i.e. persons) are able to spread the disease. If a network were perfectly homogeneous, there would be no preferential target for immunization; conversely, if only one node is able to infect others, targeting this node would be an exceptionally efficient strategy. In any targeted strategy, if we begin by immunizing those individuals with the highest spreading potential, the marginal impact of the first immunization is greater than the immunization of an average individual; however, as we move down the ranks one necessarily reaches the point at which there are no more higher-than-average spreading nodes to be immunized. Consequently, the more nodes a targeted strategy needs to immunize, the lesser the difference between it and a randomized strategy.

As such, the maximum possible relative benefit of a targeted strategy rests on two main factors: the amount of heterogeneity in spreading potential, and the necessary number of people that need to be immunized. The latter is partially dependent on the former, but also on factors such as the efficiency of immunization and the general infectiousness of the disease. While vaccines for COVID-19 provide excellent protection, the disease is also highly infectious. As we find that EHR still requires substantial vaccine coverage to achieve herd immunity, it is tempting to consider alternate vaccination strategies which might more precisely target the individuals responsible for a majority of infections. This is challenging for several reasons. Firstly, even if a factor in itself might be identified, such as certain co-morbidities or leisure activities, properly identifying which individuals actually belong to the corresponding group might be outside the capabilities of relevant authorities. Second, prioritizing vaccines according to 
certain types of adjustable behavior might be politically controversial, as it might be perceived as an incentive to (or reward for) engaging in such behavior. Third, super-spreading potential may also be driven by factors such as viral load [18, 19, which may be inherently unpredictable at the individual level, making it impossible to pre-determine which individuals are likely to cause the most secondary infections. With these concerns in mind, EHR has substantial advantages in that household sizes constitute a clearly definable criterion, can be identified within the capabilities of public authorities (as evidenced by available data from census and national statistics agencies in many countries $20[22$ ) and are also likely to be a less politically controversial target than more behavior-based alternatives.

While EHR does outperform the other strategies, the likely high reproductive number of current strains of COVID-19 requires extensive vaccination coverage in order to achieve herd immunity, enough that the choice of strategy has a reduced impact in terms of the herd immunity threshold. Even if surmountable, the organizational challenges of such a strategy may not justify the gains with regards to COVID-19. The benefits may be greater, however, in the event of a possible future pandemic with a lower $R$, but for which the mortality is high enough that strict public health countermeasures need to be enforced until a vaccine is developed and deployed. For such a disease, herd immunity thresholds would be lower, increasing the viability of targeted strategies such as EHR.

The difficulty with which age-dependent strategies achieve herd immunity, even with highly effective vaccines, may seem surprising at first. There are several explanations for this. Young adults and children tend to have a large amount of social contacts, 23,24] which increases their infectious potential. Social contact patterns are also in large part age-assortative(people tend to associate with people in their age group) 25, 26], and while minors have a certain amount of contact with adults (primarily family and educational staff), a substantial amount of their contacts are with other minors. In a situation in which all (or nearly all) adults are vaccinated while all minors remain unvaccinated, any infected minor would still infect as many other minors as if no adults were vaccinated.

It is important to keep in mind that $R$ is measured as an average over all individuals that actually get infected. As such, adults who would have very little spreading potential even if they were infected do not bring down the average, as they do not get sick. Consequently, starting from any unvaccinated scenario such that an average infected minor is likely to infect, on average, at least one other minor in addition to any adults they may infect, $R$ will remain above unity even with full vaccine coverage amongst the adult population, assuming all minors remain unvaccinated.

It is conceivable that a mortality-focused strategy (such as eldest-first) might achieve an acceptable mortality rate quicker than spread-focused strategies can achieve herd immunity, and would therefore allow for relieving disease control measures faster. In this case, a mortality-focused strategy would also be a preferable approach even if prevalence is low enough during vaccine deployment for the total number of deaths and severe illnesses to not be of particular concern. However, unlike the criterion of herd immunity, what constitutes a threshold for acceptable mortality rate is fundamentally a moral and political question, rather than a scientific one; therefore, any attempt to evaluate how efficiently any given strategy would achieve acceptable mortality is outside the scope of this article.

\section{Conclusion}

As mentioned in the introduction, vaccination for COVID-19 would allow for relieving disease prevention measures on the completion of at least one of two objectives: 
reduction in the per-case risk of serious illness and death to a level which is acceptable even with exponential spread of infection, or by achieving herd immunity through breaking of infection chains. In our simulations, we find that these two objectives conflict to some extent, and that a vaccination strategy focusing on the first objective by prioritizing vaccines according to descending age performs poorly with regards to the second objective.

The choice of vaccination strategy must be made on the basis of reducing total health and social costs over the duration of the epidemic. A strategy that requires more time in order to reach the objective might still be more appropriate if deaths and serious illness during this period are significantly reduced. A comprehensive cost-benefit evaluation of possible strategies involves, among other factors, weighing health costs against the time and resources required to implement it, and involves both ethical and scientific considerations.

However, in areas where other public health measures have been and remain sufficient for suppressing prevalence to a negligible or even non-existent level (such as parts of East Asia and Oceania), and where a low prevalence can be maintained until the vaccination program achieves a satisfactory coverage of the population, any possible direct health benefits from that a mortality-focused strategy become minimal as long as other measures remain effective. In such cases, the ultimate purpose of the vaccination program effectively consists in relieving the burden imposed by these other measures, for which an infection-targeted strategy such as EHR might be more suitable than mortality-targeted strategies such as eldest-first.

\section{Acknowledgments}

We thank HUNT cloud (https://www.ntnu.edu/mh/huntcloud) for computing resources.

\section{References}

1. McKee M. A European roadmap out of the covid-19 pandemic. BMJ. 2020;369. doi:10.1136/bmj.m1556.

2. Dooling K, McClung N, Chamberland M, Marin M, Wallace M, Bell BP, et al. The Advisory Committee on Immunization Practices' interim recommendation for allocating initial supplies of COVID-19 vaccine - United States, 2020. Morbidity and mortality weekly report. 2020;69(49):1857.

3. for Disease Prevention EC, Control. COVID-19 Vaccination and Prioritisation Strategies in the EU/EEA; 2020.

4. Thompson MG, Burgess JL, Naleway AL, Tyner HL, Yoon SK, Meece J, et al. Interim estimates of vaccine effectiveness of BNT162b2 and mRNA-1273 COVID-19 vaccines in preventing SARS-CoV-2 infection among health care personnel, first responders, and other essential and frontline workers - eight US locations, December 2020-March 2021. Morbidity and Mortality Weekly Report. 2021;70(13):495.

5. Davies NG, Abbott S, Barnard RC, Jarvis CI, Kucharski AJ, Munday JD, et al. Estimated transmissibility and impact of SARS-CoV-2 lineage B.1.1.7 in England. Science. 2021;372(6538).

6. Leung K, Shum MH, Leung GM, Lam TT, Wu JT. Early transmissibility assessment of the N501Y mutant strains of SARS-CoV-2 in the United Kingdom, 
October to November 2020. Eurosurveillance. 2021;26(1). doi:https://doi.org/10.2807/1560-7917.ES.2020.26.1.2002106.

7. Fort H. A very simple model to account for the rapid rise of the British variant of SARS-CoV-2 in several countries and the world. medRxiv. 2021;.

8. Voigt A, Martyushenko N, Karlsen E, Hall M, Nyhamar K, Omholt SW, et al. Containing pandemics through targeted testing of households. BMC infectious diseases. 2021;21(1):1-10.

9. Oliver SE, Gargano JW, Scobie H, Wallace M, Hadler SC, Leung J, et al. The advisory committee on immunization practices' interim recommendation for use of Janssen COVID-19 vaccine - United States, February 2021. Morbidity and Mortality Weekly Report. 2021;70(9):329.

10. Oliver SE. The advisory committee on immunization practices' interim recommendation for use of moderna COVID-19 vaccine - United States, December 2020. MMWR Morbidity and mortality weekly report. 2020;69.

11. Oliver SE, Gargano JW, Marin M, Wallace M, Curran KG, Chamberland M, et al. The advisory committee on immunization practices' interim recommendation for use of Pfizer-BioNTech COVID-19 vaccine-United States, December 2020. Morbidity and Mortality Weekly Report. 2020;69(50):1922.

12. Data from Norwegian Surveillance System for Communicable Disease (MSIS), and Norwegian Institute of Public Health (FHI), for the 30 weeks from March 24th - September 27th; 2020.

13. Scientific Advisory Group for Emergencies (SAGE), at SAGE 70 meeting, Paper "Housing, household transmission and ethnicity, 26 November 2020"; 2020. URL https://www.gov.uk/government/publications/housing-household-transmissionand-ethnicity-26-november-2020.

14. Pastor-Satorras R, Vespignani A. Immunization of complex networks. Phys Rev E. 2002;65:036104.

15. Dezső Z, Barabási AL. Halting viruses in scale-free networks. Phys Rev E. 2002;65:055103.

16. Cohen R, Havlin S, ben Avraham D. Efficient Immunization Strategies for Computer Networks and Populations. Phys Rev Lett. 2003;91:247901. doi:10.1103/PhysRevLett.91.247901.

17. Gómez-Gardenes J, Echenique P, Moreno Y. Immunization of real complex communication networks. The European Physical Journal B-Condensed Matter and Complex Systems. 2006;49(2):259-264.

18. Avadhanula V, Nicholson EG, Ferlic-Stark L, Piedra FA, Blunck BN, Fragoso S, et al. Viral Load of Severe Acute Respiratory Syndrome Coronavirus 2 in Adults During the First and Second Wave of Coronavirus Disease 2019 Pandemic in Houston, Texas: The Potential of the Superspreader. The Journal of Infectious Diseases. 2021;223(9):1528-1537. doi:10.1093/infdis/jiab097.

19. Goyal A, Reeves DB, Cardozo-Ojeda EF, Schiffer JT, Mayer BT. Viral load and contact heterogeneity predict SARS-CoV-2 transmission and super-spreading events. Elife. 2021;10:e63537. 
20. French National Institute of Statistics and Economic Studies - Households according to size in $2017 ; 2017$. URL

http://www.alisse2.insee.fr/fr/statistiques/2012714.

21. Statistics Norway; 2020. URL https://www.ssb.no/en/befolkning.

22. US Census Bureau; 2021. URL

https://www.census.gov/quickfacts/fact/table/US/PST045219.

23. Mossong J, Hens N, Jit M, Beutels P, Auranen K, Mikolajczyk R, et al. Social Contacts and Mixing Patterns Relevant to the Spread of Infectious Diseases. PLOS Medicine. 2008;5(3):1-1. doi:10.1371/journal.pmed.0050074.

24. Del Valle SY, Hyman JM, Hethcote HW, Eubank SG. Mixing patterns between age groups in social networks. Social Networks. 2007;29(4):539-554. doi:https://doi.org/10.1016/j.socnet.2007.04.005.

25. Prem K, Cook AR, Jit M. Projecting social contact matrices in 152 countries using contact surveys and demographic data. PLOS Computational Biology. 2017;13(9):1-21. doi:10.1371/journal.pcbi.1005697.

26. Glass LM, Glass RJ. Social contact networks for the spread of pandemic influenza in children and teenagers. BMC public health. 2008;8(1):1-15. 